\title{
Clasificación de instituciones prestadores de servicios de salud según el sistema de cuentas de la salud de la Organización para la Cooperación y el Desarrollo Económico: el caso de Colombia*
}

\section{Classification of Health Services Providing Institutions According to the System of Health Accounts of the Organization for Economic Cooperation and Development: the Colombia Case}

\section{Classificação de instituições prestadoras de serviços de saúde de acordo ao sistema de contas da saúde da Organização para a Cooperação e o Desenvolvimento Económico: 0 caso da Colômbia}

Fecha de recepción: 17-01-2017 Fecha de aceptación: 14-03-2017 Disponible en línea: 30-05-2017 doi:10.11144/Javeriana.rgps16-32.cips

Cómo citar este artículo:

Prada-Ríos SI, Pérez-Castaño AM, Rivera-Triviño AF. Clasificación de instituciones prestadores de servicios de salud según el sistema de cuentas de la salud de la Organización para la Cooperación y el Desarrollo Económico: el caso de Colombia. Rev Gerenc Polít Salud. 2017; 16 (32): 51-65. https://doi. org/10.11144/Javeriana.rgps16-32.cips

\author{
Sergio Iván Prada-Ríos** \\ Ana Melissa Pérez-Castaño** \\ Andrés Felipe Rivera-Triviño****
}

Artículo de investigación. La correspondencia sobre este artículo deberá ser enviada a Universidad Icesi, Calle 18 No. 122-135 Pance, Casa Rocha 2do piso, Cali, Colombia.

** Investigador, Ph. D. en Políticas Públicas de la Universidad de Maryland, Estados Unidos. Director del Centro de Estudios en Protección Social y Economía de la Salud (Proesa) y profesor del Departamento de Economía de la Universidad Icesi. Correo electrónico: siprada@proesa.org.co

*** Investigadora, magíster en Economía de la Universidad del Valle. Investigadora junior del Centro de Estudios en Protección Social y Economía de la Salud (Proesa), Universidad Icesi. Correo electrónico: amperez@proesa.org.co

**** Investigador, magíster en Economía de la Universidad Icesi. Estudiante de doctorado en Economía en la Facultad de Economía del Colegio Mayor de Nuestra Señora del Rosario. Correo electrónico: afrivera@icesi.edu.co 


\section{Resumen}

En Colombia las instituciones prestadoras de servicios de salud (IPS) públicas tienen una clasificación según tres niveles de complejidad: bajo, medio y alto. Esto no sucede con las IPS privadas. Esta carencia dificulta los análisis comparativos entre prestadores privados. Para suplir esta necesidad se desarrolló un algoritmo de clasificación de IPS de acuerdo con la metodología del Sistema de Cuentas de la Salud utilizado en los países de la Organización para la Cooperación y el Desarrollo Económico. Se utilizó como fuente de información la base de datos de servicios habilitados del Registro Especial de Prestadores para 2015. Los resultados muestran que en el país el $66 \%$ de las IPS son de atención ambulatoria, el 17\% hospitales, el $11 \%$ centros de promoción y prevención y el $6 \%$ prestadores de servicios auxiliares. La concentración de activos es opuesta, pues se encontró que los hospitales tienen el $90.5 \%$ de los activos.

Palabras clave: taxonomía; prestación de atención de salud; servicios de salud, sistemas de salud; instituciones de atención ambulatoria; hospitales

\section{Abstract}

In Colombia, public health service providers (IPS) are classified according to three complexity levels: low, medium, and high. Such is not the case with private IPS. This lack hinders comparative analyzes between private providers. To meet this need, an IPS classification algorithm was developed according to the methodology of the System of Health Accounts used in the member countries of the Organization for Economic Cooperation and Development. The database of qualified services of the Special Registry of Providers for 2015 was used as a source of information. The results show that $66 \%$ of the IPS in the country work in ambulatory care, $17 \%$ are hospitals, $11 \%$ are promotion and prevention centers, and $6 \%$ are ancillary service providers. The concentration of assets follows the opposite distribution, as it was found that hospitals have $90.5 \%$ of assets.

Keywords: taxonomy; provision of health care; health services, health systems; ambulatory care institutions; hospitals

\section{Resumo}

Na Colômbia as instituições prestadoras de serviços de saúde (IPS) públicas tem classificação segundo três níveis de complexidade: baixo, meio e alto. Isso não acontece com as IPS privadas. Esta carência dificulta as análises comparativas entre prestadores privados. Para suprir tal necessidade desenvolveu-se um algoritmo de classificação de IPS de acordo com a metodologia do Sistema de Contas da Saúde utilizado nos países da Organização para a Cooperação e o Desenvolvimento Econômico. Utilizou-se como fonte de informação a base de dados de serviços habilitados do Registro Especial de Prestadores para 2015. Os resultados mostram que no país $66 \%$ das IPS são de atenção ambulatorial, 17\% hospitais, o $11 \%$ centros de promoção e prevenção e $6 \%$ prestadores de serviços auxiliares. A concentração de ativos é oposta, pois se encontrou que os hospitais têm o $90.5 \%$ dos ativos.

Palavras-chave: taxonomia; prestação de atenção de saúde; serviços de saúde, sistemas de saúde; instituições de atenção ambulatorial; hospitais 
CLASIFICACIÓN DE INSTITUCIONES PRESTADORES DE SERVICIOS DE SALUD SEGÚN EL SISTEMA

DE CUENTAS DE LA SALUD DE LA ORGANIZACIÓN PARA LA COOPERACIÓN Y EL DESARROLLO

ECONÓMICO: EL CASO DE COLOMBIA

\section{Introducción}

De acuerdo con el Registro Especial de Prestadores de Salud (REPS) de Colombia, existen cuatro categorías de clasificación para los prestadores: profesionales independientes, entidades con objeto social diferente a la prestación de servicios, instituciones prestadores de servicios de salud (IPS) y transporte especial de pacientes (1).

Las IPS tienen como objeto social la prestación de servicios de salud y además se encuentran habilitadas por el Sistema Obligatorio de Garantía de Calidad (SOGC) (Resolución 1441 de 2013). En Colombia, las IPS se han clasificado, de acuerdo con su naturaleza jurídica, en públicas, privadas y mixtas. Solo para las públicas existe una categorización de acuerdo con el nivel complejidad de la institución (1).

La clasificación de prestadores por niveles de complejidad nace con la Ley 10 del 10 de enero de 1990, en la cual se establecieron responsabilidades a municipios y departamentos en la prestación de servicios, de acuerdo con tres niveles de atención. En el primer nivel, la ley, de manera genérica, clasificó a hospitales locales, centros y puestos de salud; en el segundo y tercero, los hospitales regionales, universitarios y especializados. Posteriormente, el Decreto 1760 del 2 de agosto de 1990, que reglamentó la mencionada ley, fijó los criterios para definir el grado de complejidad. Así, las entidades de primer nivel ofrecían atención por personal general, técnico y auxiliar, tecnología de baja complejidad y cobertura de atención tanto a la población del municipio como a la de aquellos municipios cercanos que no tuvieran cobertura. Las entidades de segundo nivel ofrecían atención por personal especializado, tecnología de mediana complejidad y cubrían poblaciones con uno o más municipios que gozaran del primer nivel de atención.

Asimismo, las instituciones de tercer nivel contaban con personal especializado y subespecializado, tecnología de la más alta complejidad y cobertura de segundo y tercer nivel de atención. Esta caracterización presentada corresponde a condiciones propias de la institución, y no se utilizó para clasificar las IPS privadas. Además, esta metodología no tiene en cuenta, de manera directa, la cantidad o clase de servicios prestados, dejando de lado un aspecto muy importante para diferenciar a los prestadores de salud.

Posteriormente, con la Resolución 1043 y el Decreto 1011 del 3 de abril de 2006, se establecieron las condiciones que deben cumplir los prestadores públicos y privados para habilitar sus servicios, indicándose los niveles de complejidad como un atributo de cada servicio habilitado y no de la institución.

Así, en Colombia las IPS públicas tienen una clasificación según tres niveles, mientras que las IPS privadas no. A la fecha no existe ninguna taxonomía en el país que clasifique todas las IPS, públicas o privadas, de acuerdo con los servicios prestados. Esto dificulta la posibilidad de realizar análisis de tipo comparativo, por ejemplo, en el ámbito financiero o en el de calidad. En Colombia solo existe un estudio (1) publicado con clasificación de IPS, y en este se utilizan las categorías del REPS; por ejemplo, la razón social: públicas, privadas o mixtas. Esta clasificación deja de lado aspectos relevantes en la estructura como su tamaño y funcionalidad (véase figura 1). 


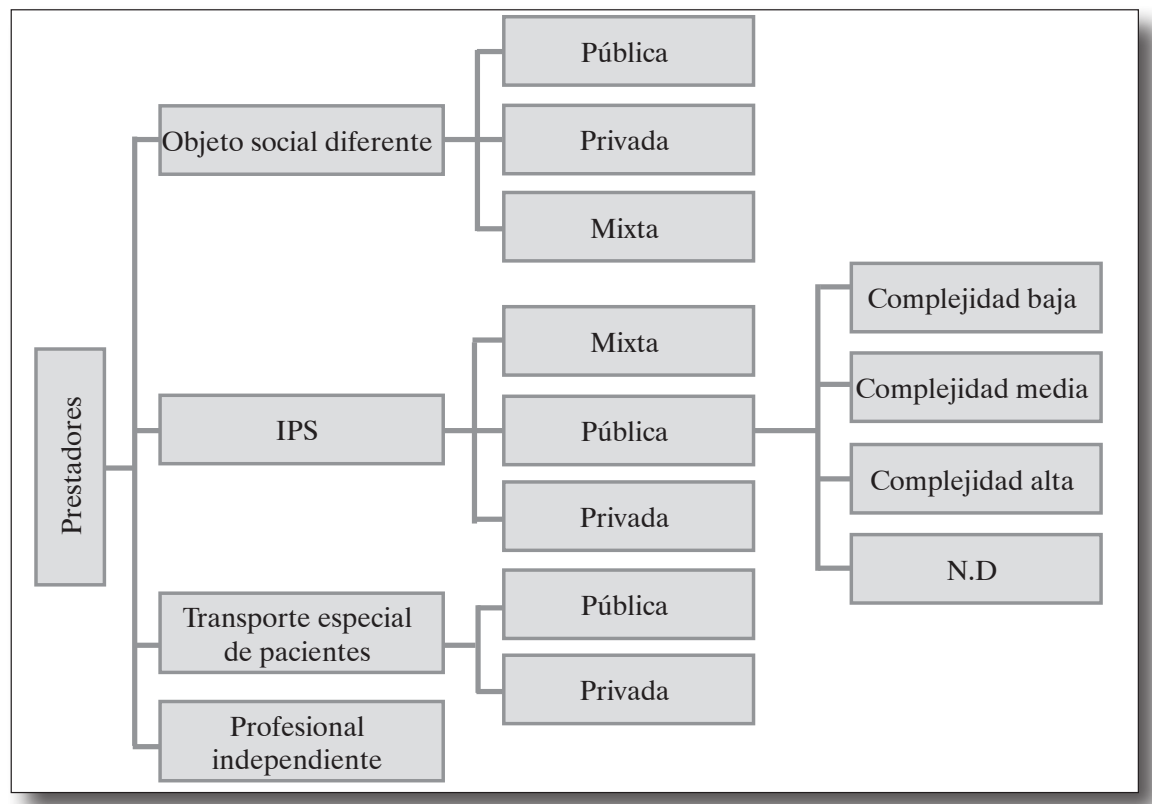

Figura 1. TAXonomía de PRESTADORES de ACUERdo CON EL REPS

Fuente: Registro Especial de Prestadores (1)

El objetivo de esta investigación es desarrollar un algoritmo clasificador de IPS, públicas o privadas, de acuerdo con el tipo de servicios prestados por la institución. Se emplea la estructura de clasificación de prestadores del Sistema de Cuentas de Salud (SCS) del año 2011 (véase tabla 1), promovido por la Organización para la Cooperación y el Desarrollo Económico (OCDE) como marco conceptual para la estandarización de la prestación de servicios médicos de salud. Se utiliza como fuente de información y unidad de clasificación los servicios habilitados para cada una de las instituciones en el REPS.

Para demostrar la utilidad analítica de la clasificación obtenida se utilizan los reportes de circular única (CU) y del Sistema de Información de Hospitales Públicos (SIHO) para estimar el tamaño financiero del sector prestador según su nivel de activos y las diferentes categorías del SCS.
Este documento está conformado por cuatro secciones. La primera de ellas es esta introducción, la segunda presenta la metodología del estudio, la tercera muestra los resultados obtenidos y la cuarta concluye.

\section{Materiales y métodos}

El SCS inició en el año 2000 con el objetivo de estructurar las cuentas entre países y poder así hacer estudios comparativos. Esta metodología también es usada para definir categorías de la atención en salud, proveer información para estudios financieros, realizar informes secuenciales a través del tiempo e integrar análisis del tipo meso y micro en la atención en salud (2).

La importancia de esta metodología está en la posibilidad que ofrece de compararse con los países pertenecientes a la OCDE, e incluso con otros que también la utilizan. 
Sin embargo, es claro que para poder usarla en países con características y fuentes de información disimiles, es necesario realizar adaptaciones. Por ejemplo el mismo SCS incluye la guía del productor de cuentas nacionales de salud, enfocada en los países de ingresos medios y bajos, con una visión más amplia del gasto en salud, incluyendo fomento y restauración o mantenimiento (3).

La versión más actual del SCS fue publicada en el año 2011 y guarda similitud con los objetivos inicialmente planteados (4). Dicha versión es la que se utiliza en este estudio.

\section{Definiciones del SCS}

De acuerdo con el SCS, existen nueve categorías en las cuales se puede clasificar un prestador: hospitales, cuidado residencial de largo plazo, prestadores de atención ambulatoria, servicios auxiliares, minoristas y otros prestadores de bienes médicos, prestadores para el cuidado preventivo, para la administración y financiación del sistema de salud, el resto de la economía y del mundo. Las categorías relacionadas con otros actores del sistema de salud, como hogares (resto de la economía), aseguradores (administración y financiación del sistema de salud) y prestadores no residentes del país (resto del mundo), no forman parte de esta investigación, ya que el objetivo de este trabajo se centra en los prestadores de servicios de salud cuya información proviene del REPS. Para conocer en detalle la descripción de estas tres categorías excluidas remítase al SCS del año 2011.

A continuación, se exponen las definiciones de las seis categorías empleadas en la clasificación:

Hospitales: son establecimientos que tienen como actividad principal la prestación, el diagnóstico y el tratamiento de servicios médicos. Aunque su prioridad es la parte hospitalaria también prestan servicios no hospitalarios. De acuerdo con el tratamiento médico y la especificidad de la enfermedad, los hospitales pueden clasificarse en: generales, de salud mental y especializados.

Cuidado residencial de largo plazo: son establecimientos que tienen como actividad principal el cuidado de personas en un largo plazo, incluyendo servicios de enfermería y otro tipo de supervisión que requieran los hospitalizados. Los servicios prestados normalmente son una mezcla entre salud y servicio social, con menos intensidad médica que un hospital. Dentro de esta categoría se encuentran centros médicos: de cuidado en largo plazo, de salud mental y abuso de sustancias y otros centros residenciales.

Prestadores de servicio ambulatorio: comprende aquellos establecimientos que se encargan de la prestación de servicios de salud para personas que no requieren un servicio hospitalario. Se incluyen tanto la atención en un consultorio médico como la visita de un profesional a la casa del paciente. Dentro de los prestadores ambulatorios se encuentran: consultorios médicos (generales, de salud mental y especialistas), odontológicos, de otros profesionales de la salud, centros de cuidado ambulatorio (planificación familiar, salud mental y adicciones, cirugía ambulatoria, diálisis y otros ambulatorios) y prestadores de servicios de salud en casa.

Prestadores de servicios auxiliares: se caracterizan por brindar servicios auxiliares a los pacientes no hospitalizados, mediante la supervisión de un profesional de la salud. Forman parte de esta categoría prestadores de transporte de pacientes (servicios de ambulancias para pacientes con o sin emergencia médica), laboratorios médicos y de diagnóstico (centros de imágenes diagnósticas, laboratorios de rayos x y laboratorios de patologías) y otros prestadores de servicios 
auxiliares (servicios auxiliares que no se incluyeron en las categorías previas).

Prestadores de promoción y prevención: está conformado por prestadores que se encargan de realizar programas de promoción, campañas de salud pública para grupos específicos de individuos o población en general. Estos servicios son realizados por agencias de promoción y prevención en salud, institutos de salud pública, etcétera.

\section{Aproximación al SCS}

Para realizar la clasificación se utilizó como fuente de información los servicios habilitados que reportan las IPS al REPS. Dado que el REPS es una fuente de información dinámica, que no guarda registro histórico, es importante aclarar que, a efectos de este ejercicio, se utilizó la base de datos de servicios del mes de abril de 2015. La lógica del algoritmo es distribuir las IPS en categorías que tengan características comunes, determinadas por la cantidad de servicios habilitados. Los servicios pueden ser misionales o complementarios. Por misionales se entiende servicios principales o acordes al objeto de la entidad y por complementarios servicios secundarios o de apoyo en la prestación.
En la estructuración de cada categoría se empleó como criterio de selección que cada IPS tuviese más del $50 \%$ de los servicios habilitados en determinado grupo, teniendo en cuenta que una entidad debe tener al menos un servicio misional. Por ejemplo, una institución que se dedica a la odontología tiene tres servicios: odontología general, esterilización y toma e interpretación de radiologías. En este caso uno de los tres servicios habilitados es odontológico, sin embargo, no alcanza a sobrepasar el $50 \%$, pero es evidente que los servicios complementarios no identifican a esta IPS en otra categoría y además sirven en la prestación de los servicios odontológicos. Por tanto, en este caso, esta IPS se clasificaría en prestadores de atención ambulatoria - consultorios odontológicos.

Es preciso documentar que la unidad de análisis en este caso es la IPS y no la sede de la IPS, de tal forma que la unidad de análisis está determinada por el número de identificación tributaria (NIT) de cada entidad.

En la tabla 1 se muestran las seis categorías establecidas y la forma como se adoptó el concepto del SCS a los servicios habilitados en el REPS.

Tabla 1. Taxonomía Sistema de Cuentas de Salud (SHA)

\begin{tabular}{|l|l|l|}
\hline \multicolumn{1}{|c|}{ Categoría } & \multicolumn{1}{|c|}{ Subcategoría } & \multicolumn{1}{c|}{ Definición } \\
\hline HP1: & $\begin{array}{l}\text { Se dedican a brindar diagnóstico general y tratamiento } \\
\text { médico a pacientes hospitalizados con diversas condiciones } \\
\text { médicas. También prestan otros servicios no hospitalarios } \\
\text { como: patologías, diagnóstico de rayos X, laboratorio clínico, } \\
\text { quirófanos o farmacias, los cuales se utilizan tanto para } \\
\text { pacientes hospitalizados como para los ambulatorios. Hospi- } \\
\text { tales universitarios, de enseñanza y para las fuerzas públicas } \\
\text { son ejemplos de hospitales generales. Criterio de selección: } \\
\text { prestadores con al menos tres servicios hospitalarios misiona- } \\
\text { les (generales o de cuidados intensivos). }\end{array}$ \\
\hline
\end{tabular}




\section{CLASIFICACIÓN DE INSTITUCIONES PRESTADORES DE SERVICIOS DE SALUD SEGÚN EL SISTEMA DE CUENTAS DE LA SALUD DE LA ORGANIZACIÓN PARA LA COOPERACIÓN Y EL DESARROLLO ECONÓMICO: EL CASO DE COLOMBIA}

\begin{tabular}{|c|c|c|}
\hline Categoría & Subcategoría & Definición \\
\hline \multirow[t]{2}{*}{$\begin{array}{l}\text { HP1: } \\
\text { Hospitales }\end{array}$} & $\begin{array}{l}\text { HP1.2. } \\
\text { Hospitales de } \\
\text { salud mental y } \\
\text { adicciones }\end{array}$ & $\begin{array}{l}\text { Realizan diagnóstico, tratamiento médico y monitoreo de } \\
\text { pacientes con enfermedades mentales o problemas asociadas } \\
\text { al abuso de sustancias. Como actividad secundaria prestan } \\
\text { servicios de exámenes de laboratorio clínico, diagnóstico } \\
\text { de rayos X y servicios de encefalograma. Los hospitales psi- } \\
\text { quiátricos y los de abuso de sustancias son un ejemplo de la } \\
\text { categoría de salud mental. Criterio de selección: prestadores } \\
\text { dedicados principalmente a servicios hospitalarios en salud } \\
\text { mental y psiquiatría. }\end{array}$ \\
\hline & $\begin{array}{l}\text { HP.1.3. } \\
\text { Hospitales de } \\
\text { especialidades }\end{array}$ & $\begin{array}{l}\text { Prestan servicios de diagnóstico, tratamiento médico y } \\
\text { monitoreo a pacientes con una condición o enfermedad } \\
\text { específica. Un ejemplo de esta categoría son los hospitales } \\
\text { cardiológicos. Criterio de selección: prestadores con al } \\
\text { menos un servicio hospitalario misional habilitado y que no } \\
\text { se identifican como hospital general ni mental; por ejemplo, } \\
\text { hospitales pediátricos. }\end{array}$ \\
\hline \multirow{3}{*}{$\begin{array}{l}\text { HP2: } \\
\text { Establecimientos } \\
\text { de cuidado } \\
\text { residencial de } \\
\text { largo plazo }\end{array}$} & $\begin{array}{l}\text { HP2.1. } \\
\text { Centros } \\
\text { residenciales de } \\
\text { cuidado médico a } \\
\text { largo plazo }\end{array}$ & $\begin{array}{l}\text { Prestan servicios de enfermería y rehabilitación para pacien- } \\
\text { tes hospitalizados por un largo periodo. También ofrecen } \\
\text { servicios de apoyo social como, por ejemplo, asistencia en } \\
\text { tareas cotidianas. Los hospitales o casas para convalecientes } \\
\text { y las casas para adultos mayores son un ejemplo de centros } \\
\text { médicos de cuidado a largo plazo. } \\
\text { Criterio de selección: prestadores que proveen servicios de } \\
\text { enfermería y rehabilitación mediante la hospitalización. }\end{array}$ \\
\hline & $\begin{array}{l}\text { HP2.2. Centros } \\
\text { de salud mental } \\
\text { y abuso de } \\
\text { sustancias }\end{array}$ & $\begin{array}{l}\text { Proveen, en un ambiente de hospitalización, servicios } \\
\text { domiciliarios para personas con problemas mentales. Se } \\
\text { enfocan en brindar atención en alimentación y vivienda, con } \\
\text { supervisión y asesoramiento. Residencias para personas con } \\
\text { retardos mentales, centros de rehabilitación de alcohólicos o } \\
\text { de personas con adicciones a las drogas forman parte de esta } \\
\text { categoría. Criterio de selección: prestadores que ofrecen ser- } \\
\text { vicios de hospitalización a pacientes con problemas mentales } \\
\text { y abuso de sustancias psicoactivas. }\end{array}$ \\
\hline & $\begin{array}{l}\text { HP2.9. Otros } \\
\text { establecimientos } \\
\text { residenciales de } \\
\text { cuidado largo } \\
\text { plazo }\end{array}$ & $\begin{array}{l}\text { Ofrecen servicios para el cuidado de la salud en el largo pla- } \\
\text { zo, no incluidos en ninguna de las dos categorías anteriores. } \\
\text { Criterio de selección: otros prestadores que ofrecen servicios } \\
\text { de hospitalización (se cuentan aquellos que combinan los dos } \\
\text { servicios anteriores). }\end{array}$ \\
\hline \multirow[t]{2}{*}{$\begin{array}{l}\text { HP3: } \\
\text { Prestadores de } \\
\text { atención } \\
\text { ambulatoria }\end{array}$} & $\begin{array}{l}\text { HP3.1. } \\
\text { Consultorios } \\
\text { médicos }\end{array}$ & $\begin{array}{l}\text { Se incluyen médicos generales y especialistas que se dedican } \\
\text { de manera independiente a prestar servicios de medicina } \\
\text { general o especializada, en oficinas propias o alquiladas, en } \\
\text { clínicas o centros que no forman parte de un hospital u orga- } \\
\text { nización. Los consultorios médicos se clasifican en: generales, } \\
\text { de salud mental y especialistas. } \\
\text { Criterio de selección: prestadores que ofrecen servicios de } \\
\text { consulta externa general o especializada. }\end{array}$ \\
\hline & $\begin{array}{l}\text { HP3.1.1. } \\
\text { Consultorios } \\
\text { medicina general }\end{array}$ & $\begin{array}{l}\text { Establecimientos de profesionales que se dedican principal- } \\
\text { mente a prestar servicios de medicina general. Los centros o } \\
\text { consultorios médicos privados generales o de familia forman } \\
\text { parte esta categoría. Criterio de selección: consultorios } \\
\text { médicos que prestan, exclusivamente, servicios de consulta } \\
\text { externa general. }\end{array}$ \\
\hline
\end{tabular}




\begin{tabular}{|c|c|c|}
\hline Categoría & Subcategoría & Definición \\
\hline \multirow{7}{*}{$\begin{array}{l}\text { HP3: } \\
\text { Prestadores de } \\
\text { atención } \\
\text { ambulatoria }\end{array}$} & $\begin{array}{l}\text { HP3.1.2. } \\
\text { Consultorios de } \\
\text { especialistas en } \\
\text { salud mental }\end{array}$ & $\begin{array}{l}\text { Establecimientos manejados por profesionales indepen- } \\
\text { dientes que tienen una especialidad en medicina mental o } \\
\text { una cualificación correspondiente. Los psiquiatras indepen- } \\
\text { dientes y los consultorios en salud mental son ejemplo de } \\
\text { este grupo. Criterio de selección: consultorios médicos que } \\
\text { exclusivamente prestan servicios en salud mental (misional- } \\
\text { mente psiquiatría). }\end{array}$ \\
\hline & $\begin{array}{l}\text { HP3.1.3. } \\
\text { Consultorios } \\
\text { de médicos } \\
\text { especialistas }\end{array}$ & $\begin{array}{l}\text { Comprende los establecimientos manejados por profesio- } \\
\text { nales independientes que tienen una especialidad médica } \\
\text { diferente a salud mental y medicina general. Criterio de } \\
\text { selección: consultorios médicos que prestan servicios de con- } \\
\text { sulta externa especializada (excluyendo salud mental). Las } \\
\text { especialidades son: banco de sangre, anestesiología, endocri- } \\
\text { nología, geriatría, infectología, patología, del deporte, de tra- } \\
\text { bajo, medicina interna, otorrinolaringología, reumatología, } \\
\text { inmunología, angiología, cirugía torácica, cirugía de mano, } \\
\text { de cabeza y cuello, cirugía general, radioterapia, nefrología, } \\
\text { oftalmología, rehabilitación, medicina nuclear, medicina } \\
\text { estética, dermatología, gastroenterología, hematología, neu- } \\
\text { mología, neurología, traumatología, urología, ginecología, } \\
\text { medicina alternativa, cardiología, pediatría, oncología, varias } \\
\text { especialidades y otras especialidades. }\end{array}$ \\
\hline & $\begin{array}{l}\text { HP3.2. } \\
\text { Consultorios } \\
\text { odontológicos }\end{array}$ & $\begin{array}{l}\text { Establecimientos manejados por profesionales independien- } \\
\text { tes que tienen un título de médico dental u otra cualificación } \\
\text { correspondiente, como dentistas, ortodontistas, endodoncis- } \\
\text { tas, cirujanos orales o maxilofaciales. Criterio de selección: } \\
\text { consultorios que prestan servicios de consulta externa y } \\
\text { cirugía ambulatoria para el cuidado dental. }\end{array}$ \\
\hline & $\begin{array}{l}\text { HP3.3. } \\
\text { Consultorios } \\
\text { de otros } \\
\text { profesionales de } \\
\text { la salud }\end{array}$ & $\begin{array}{l}\text { Están conformados por médicos y paramédicos independien- } \\
\text { tes que proveen servicios ambulatorios y no se encuentran } \\
\text { clasificados en las categorías anteriores. Criterio de selección: } \\
\text { consultorios que prestan servicios de consulta externa y } \\
\text { apoyo diagnóstico y terapéutico en: enfermería, fisioterapia, } \\
\text { fonoaudiología, nutrición y dietética, salud ocupacional, tera- } \\
\text { pia ocupacional, terapia respiratoria y atención preventiva en } \\
\text { salud e higiene oral. }\end{array}$ \\
\hline & $\begin{array}{l}\text { HP3.4. } \\
\text { Centros de } \\
\text { atención } \\
\text { ambulatoria }\end{array}$ & $\begin{array}{l}\text { Son establecimientos que proveen una gran variedad de } \\
\text { servicios ambulatorios (de consulta externa) por medio de un } \\
\text { grupo de médicos o paramédicos. Criterio de selección: cen- } \\
\text { tros de atención ambulatoria dedicados a servicios de salud } \\
\text { mental y adicciones, cirugía ambulatoria y diálisis. }\end{array}$ \\
\hline & $\begin{array}{l}\text { HP3.4.1. } \\
\text { Centros de } \\
\text { planificación } \\
\text { familiar }\end{array}$ & $\begin{array}{l}\text { Estos establecimientos están conformados por un equipo } \\
\text { médico o paramédico que provee servicios ambulatorios de } \\
\text { planificación familiar, tales como: servicios de anticoncep- } \\
\text { ción, asesoramiento prenatal y esterilización. Criterio de } \\
\text { selección: centros de atención dedicados, principalmente, a la } \\
\text { promoción y prevención en planificación familiar. }\end{array}$ \\
\hline & $\begin{array}{l}\text { HP3.4.2. } \\
\text { Centros de } \\
\text { salud mental y } \\
\text { adicciones }\end{array}$ & $\begin{array}{l}\text { Establecimientos conformados por un equipo médico o } \\
\text { paramédico que proveen servicios ambulatorios relacionados } \\
\text { con el tratamiento y diagnóstico en salud mental y abuso } \\
\text { de sustancias. Criterio de selección: centros de atención en } \\
\text { drogadicción ambulatoria y unidades de rehabilitación. }\end{array}$ \\
\hline
\end{tabular}




\section{CLASIFICACIÓN DE INSTITUCIONES PRESTADORES DE SERVICIOS DE SALUD SEGÚN EL SISTEMA DE CUENTAS DE LA SALUD DE LA ORGANIZACIÓN PARA LA COOPERACIÓN Y EL DESARROLLO ECONÓMICO: EL CASO DE COLOMBIA}

\begin{tabular}{|c|c|c|}
\hline Categoría & Subcategoría & Definición \\
\hline \multirow{5}{*}{$\begin{array}{l}\text { HP3: } \\
\text { Prestadores de } \\
\text { atención } \\
\text { ambulatoria }\end{array}$} & $\begin{array}{l}\text { HP3.4.3. } \\
\text { Centros } \\
\text { independientes } \\
\text { de cirugía } \\
\text { ambulatoria }\end{array}$ & $\begin{array}{l}\text { Establecimientos especializados conformados por un grupo } \\
\text { de médicos en la prestación de servicios quirúrgicos ambu- } \\
\text { latorios. Criterio de selección: centros dedicados a cirugías } \\
\text { ambulatorias de cabeza y cuello, cardiovascular, general, } \\
\text { ginecológica, neurológica, ortopédica, oftalmológica, otorri- } \\
\text { nolaringológica, oncológica, pediátrica, plástica y estética, } \\
\text { vascular y angiológica, urológica, neurológica, torácica, der- } \\
\text { matológica, gastrointestinal, de pulmón, de mano, de mama, } \\
\text { de piel y de tejidos cardiovasculares. }\end{array}$ \\
\hline & $\begin{array}{l}\text { HP3.4.4. Centros } \\
\text { de diálisis }\end{array}$ & $\begin{array}{l}\text { Establecimientos conformados por un grupo de médicos que } \\
\text { prestan servicios de atención ambulatoria de diálisis renal o } \\
\text { de riñón. Criterio de selección: centros de apoyo diagnóstico } \\
\text { y complementación terapéutica dedicados a la nefrología, la } \\
\text { hemodiálisis y la diálisis peritoneal. }\end{array}$ \\
\hline & $\begin{array}{l}\text { HP3.4.9. } \\
\text { Otros centros } \\
\text { ambulatorios }\end{array}$ & $\begin{array}{l}\text { Establecimientos conformados por un grupo de médicos y } \\
\text { paramédicos que brindan servicios ambulatorios en diversas } \\
\text { especialidades. Criterio de selección: centros que combinan } \\
\text { la prestación de servicios de planificación familiar, de salud } \\
\text { mental, de cirugías y de diálisis. }\end{array}$ \\
\hline & $\begin{array}{l}\text { HP3.5. } \\
\text { Prestadores de } \\
\text { servicios de salud } \\
\text { en casa }\end{array}$ & $\begin{array}{l}\text { Establecimientos que prestan servicios en enfermería de } \\
\text { manera domiciliaria. Además, proveen otros servicios de } \\
\text { atención personalizada como: apoyo en los medicamentos, } \\
\text { uso de equipos, terapia ocupacional, atención a domicilio las } \\
\text { veinticuatro horas, etc. Criterio de selección: prestadores que } \\
\text { ofrecen servicios de atención domiciliaria a pacientes agudos } \\
\text { y crónicos. }\end{array}$ \\
\hline & $\begin{array}{l}\text { HP3.6. Otros } \\
\text { servicios } \\
\text { ambulatorios }\end{array}$ & $\begin{array}{l}\text { Categoría anexada al SCS. Criterio de selección: prestadores de } \\
\text { atención ambulatoria no clasificados en ninguna de las catego- } \\
\text { rías anteriores debido a que son una combinación de estos. }\end{array}$ \\
\hline \multirow{3}{*}{$\begin{array}{l}\text { HP4: } \\
\text { Prestadores } \\
\text { de servicios } \\
\text { auxiliares }\end{array}$} & $\begin{array}{l}\text { HP4.1. Transporte } \\
\text { de pacientes } \\
\text { y rescate de } \\
\text { emergencias }\end{array}$ & $\begin{array}{l}\text { Se dedican principalmente a brindar el transporte (terrestre } \\
\text { o aéreo) para pacientes que tienen una emergencia médica o } \\
\text { necesitan ser transportados, por diversas razones (transferen- } \\
\text { cia hospitalaria, transporte a pacientes con diálisis, quimiote- } \\
\text { rapia, etc.). Criterio de selección: prestadores que se dedican } \\
\text { al transporte especial de pacientes (básico o medicalizado). }\end{array}$ \\
\hline & $\begin{array}{l}\text { HP4.2. } \\
\text { Laboratorios } \\
\text { médicos y de } \\
\text { diagnóstico }\end{array}$ & $\begin{array}{l}\text { Son prestadores de servicios de diagnóstico a pacientes no } \\
\text { hospitalizados, con o sin remisión de un profesional de la sa- } \\
\text { lud. Se incluyen servicios de fluido corporal o pruebas gené- } \\
\text { ticas. Criterio de selección: prestadores que ofrecen servicios } \\
\text { de imágenes diagnósticas, rayos X (dentales o generales), } \\
\text { laboratorios clínicos, patológicos y forenses. }\end{array}$ \\
\hline & $\begin{array}{l}\text { HP4.9. Otros } \\
\text { prestadores } \\
\text { de servicios } \\
\text { auxiliares }\end{array}$ & $\begin{array}{l}\text { Dentro de esta categoría se ubican todos los prestadores de } \\
\text { servicios auxiliares que no se incluyeron en las categorías pre- } \\
\text { vias. Criterio de selección: prestadores que ofrecen servicios } \\
\text { auxiliares y no se encuentran en las dos categorías previas. }\end{array}$ \\
\hline
\end{tabular}




\begin{tabular}{|c|c|c|}
\hline Categoría & Subcategoría & Definición \\
\hline \multirow{2}{*}{$\begin{array}{l}\text { HP5: } \\
\text { Minoristas y } \\
\text { otros prestadores } \\
\text { de bienes } \\
\text { médicos }\end{array}$} & HP5.1. Farmacias & $\begin{array}{l}\text { Comprende todos los prestadores que se encargan de la } \\
\text { venta al por menor de medicamentos, con prescripción } \\
\text { médica o sin ella y están regulados. Las redes de farmacias } \\
\text { y las farmacias independientes de supermercados hacen } \\
\text { parte de esta categoría. Criterio de selección: prestadores } \\
\text { dedicados a los servicios farmacéuticos. }\end{array}$ \\
\hline & $\begin{array}{l}\text { HP5.2. } \\
\text { Vendedores al } \\
\text { por menor y otros } \\
\text { prestadores de } \\
\text { bienes médicos } \\
\text { duraderos y de } \\
\text { aparatos médicos }\end{array}$ & $\begin{array}{l}\text { Estos establecimientos se dedican a la venta al por menor } \\
\text { de bienes médicos durables y aparatos médicos tales como: } \\
\text { audífonos, lentes ópticos, prótesis, otros productos para la } \\
\text { visión, entre otros. Criterio de selección: prestadores dedica- } \\
\text { dos a los servicios ópticos. }\end{array}$ \\
\hline $\begin{array}{l}\text { HP6: } \\
\text { Prestadores de } \\
\text { promoción y } \\
\text { prevención }\end{array}$ & $\begin{array}{l}\text { HP6.1. Centros } \\
\text { de promoción y } \\
\text { prevención }\end{array}$ & $\begin{array}{l}\text { Son establecimientos encargados, principalmente, de regular } \\
\text { las actividades de las entidades prestadoras de salud y de toda } \\
\text { la administración del sector (administración y financiación en } \\
\text { salud). Es decir, dentro de esta categoría se ubican las enti- } \\
\text { dades de administración en salud del Gobierno, }{ }^{1} \text { las agencias } \\
\text { de seguridad social, }{ }^{2} \text { de administración de seguros privados }{ }^{3} \text { y } \\
\text { otras agencias de administración. Criterio de selección: centros } \\
\text { dedicados a la promoción y prevención en salud. }\end{array}$ \\
\hline
\end{tabular}

Fuente: elaboración propia con base en el SCS del año 2011 (4)

\section{Fuente de información}

Los datos utilizados provienen de tres fuentes de información: el REPS, la Superintendencia Nacional de Salud y el SIHO.

El REPS es la fuente oficial de la oferta nacional de prestadores de salud. Todo prestador, bien sea profesional independiente, IPS o una entidad con objeto social diferente al de la salud, que desee prestar un servicio en Colombia, debe estar inscrito y habilitado en este registro. Además, el proceso de habilitación se realiza por cada uno de los servicios que se presten y la complejidad que manejen. Adicionalmente, también se reporta la capacidad instalada, determinada por el número de camas, la cantidad de salas y de ambulancias. De esta fuente se incluyeron los datos de los servicios que cada prestador tiene habilitados para ofrecer al público.
La Superintendencia Nacional de Salud, como ente regulador, de inspección y vigilancia, emitió por medio de un acto administrativo la Circular Única. En este documento se da a las IPS privadas las instrucciones y normativas sobre el suministro de información. Entre los datos que se debe entregar se encuentran reportes financieros como balances y estados de resultados. Para el análisis financiero se utilizaron los datos provenientes de los balances tomados a julio de 2014. Aunque el reporte de la Superintendencia es obligatorio, la cobertura en el registro de información no es total.

Para las IPS públicas del sector se utilizaron los datos financieros del SIHO. Este sistema recopila información administrativa, financiera y técnica de las IPS públicas. La información proveniente de este sistema tiene una alta tasa de reporte en comparación con las IPS privadas.

1 Ministerio de Salud, comisiones, entre otros.

2 Administradoras de seguridad social, de seguros de accidentes y de planes voluntarios en salud.

3 Administradoras de seguros obligatorios, complementarios, fondos de seguros privados, etc. 
CLASIFICACIÓN DE INSTITUCIONES PRESTADORES DE SERVICIOS DE SALUD SEGÚN EL SISTEMA DE CUENTAS DE LA SALUD DE LA ORGANIZACIÓN PARA LA COOPERACIÓN Y EL DESARROLLO ECONÓMICO: EL CASO DE COLOMBIA

\section{Resultados}

De acuerdo con el REPS utilizado existen 8461 IPS (datos con corte al mes de abril de 2015). De estas, el 66\% se categorizó como prestadores de atención ambulatoria, con mayor participación de los consultorios médicos. A continuación se encuentran los hospitales, los cuales aportan el $17 \%$ de las instituciones. Con menor participación se ubican los centros de promoción y prevención, los prestadores de servicios auxiliares, minoristas y otros prestadores de bienes médicos, los cuales participan con 11.6, y $1 \%$, respectivamente (véase tabla 2).

Tabla 2. Clasificación SHA, RePorte y balance general a Junio de 2014, Colombia (Millones de PESOS)

\begin{tabular}{|c|c|c|c|c|c|c|}
\hline Categoría & Subcategoría ${ }^{4}$ & IPS & $\begin{array}{l}\text { IPS con reporte } \\
\text { de balance }\end{array}$ & Activos & Pasivos & Patrimonio \\
\hline \multirow{4}{*}{ HP1: Hospitales } & Total & 1404 & $1238^{5}$ & 10860735 & 2952752 & 7907984 \\
\hline & HP1.1. & 884 & 828 & 8414377 & 2493795 & 5920582 \\
\hline & HP1.2. & 24 & 15 & 77229 & 17563 & 59666 \\
\hline & HP.1.3. & 496 & 395 & 2369129 & 441393 & 1927736 \\
\hline \multirow{4}{*}{$\begin{array}{l}\text { HP2: } \\
\text { Establecimientos } \\
\text { de atención } \\
\text { medicalizada y } \\
\text { residencial }\end{array}$} & Total & 7 & 4 & 22621 & 2527 & 20094 \\
\hline & HP2.1. & 0 & 0 & & & \\
\hline & HP2.2. & 7 & 4 & 22621 & 2527 & 20094 \\
\hline & HP2.9. & 0 & 0 & & & \\
\hline \multirow{15}{*}{$\begin{array}{l}\text { HP3: } \\
\text { Prestadores } \\
\text { de atención } \\
\text { ambulatoria }\end{array}$} & Total & 5593 & 3485 & 1117130 & 361296 & 755834 \\
\hline & HP3.1. & 1607 & 1054 & 3306 & 1609 & 1697 \\
\hline & HP3.1.1. & 107 & 59 & 189 & 96 & 93 \\
\hline & HP3.1.2. & 15 & 10 & 17 & 10 & 7 \\
\hline & HP3.1.3. & 1485 & 985 & 3100 & 1502 & 1598 \\
\hline & HP3.2. & 1041 & 660 & 899 & 459 & 440 \\
\hline & HP3.3. & 1462 & 816 & 28422 & 5444 & 22978 \\
\hline & HP3.4. & 337 & 260 & 407806 & 195326 & 212480 \\
\hline & HP3.4.1. & 0 & 0 & & & \\
\hline & HP3.4.2. & 23 & 13 & 9 & 5 & 5 \\
\hline & HP3.4.3. & 243 & 192 & 383812 & 194258 & 189553 \\
\hline & HP3.4.4. & 7 & 4 & 25 & 15 & 9 \\
\hline & HP3.4.9. & 64 & 51 & 23961 & 1048 & 2913 \\
\hline & HP3.5. & 491 & 245 & 6188 & 1836 & 4352 \\
\hline & HP3.6. & 655 & 450 & 670509 & 156622 & 513887 \\
\hline \multirow{4}{*}{$\begin{array}{l}\text { P4: } \\
\text { Prestadores } \\
\text { de servicios } \\
\text { auxiliares }\end{array}$} & Total & 483 & 303 & 3135 & 935 & 2200 \\
\hline & HP4.1. & 80 & 33 & 77 & 31 & 46 \\
\hline & HP4.2. & 294 & 198 & 466 & 224 & 241 \\
\hline & HP4.9. & 109 & 72 & 2593 & 680 & 1912 \\
\hline
\end{tabular}

4 Existen 520 IPS que no lograron agruparse en la base de datos del REPS, las cuales reportan activos por \$3543 millones de pesos, pasivos por $\$ 1537$ millones de pesos y patrimonio por \$2006 millones de pesos

5 Aunque reportan 1242 IPS, se excluyeron cuatro por inconsistencias en los balances. 


\begin{tabular}{|l|l|l|c|c|c|c|}
\hline \multicolumn{1}{|c}{ Categoría } & Subcategoría $^{4}$ & IPS & $\begin{array}{c}\text { IPS con reporte } \\
\text { de balance }\end{array}$ & Activos & Pasivos & Patrimonio \\
\hline $\begin{array}{l}\text { HP5: Minoristas y } \\
\text { otros prestadores de } \\
\text { bienes médicos }\end{array}$ & Total & 70 & 43 & 89 & 41 & 48 \\
\cline { 2 - 7 } & HP5.1. & 7 & 4 & 7 & 5 & 2 \\
\cline { 2 - 7 } & HP5.2. & 63 & 39 & 82 & 36 & 46 \\
\hline $\begin{array}{l}\text { HP6: Centros } \\
\text { de promoción y } \\
\text { prevención }\end{array}$ & HP6.1. & 904 & 462 & 1710 & 647 & 1063 \\
\hline Total & & 8461 & 5535 & 12005420 & 3318197 & 8687223 \\
\hline
\end{tabular}

Fuente: Datos financieros a junio de 2014. Circular Única y Sistema de Información Hospitalario

En el interior de la categoría de prestadores de atención ambulatoria, la mayoría de las IPS pertenecen a tres subcategorías: "HP3.1.3. Consultorios de médicos especialistas" (27\%); "HP3.3 Consultorios de otros profesionales de la salud" (26\%) y "HP3.2. Consultorios odontológicos" (19\%). A su vez, dentro de la categoría hospitales, la composición muestra que el $63 \%$ son "HP1.1. Hospitales generales", el $35 \%$ son "HP.1.3. Hospitales de especialidades", y solo un restante $2 \%$ son "HP1.2. Hospitales de salud mental y adicciones".

Es importante aclarar que la poca representatividad de la categoría de atención medicalizada y residencial se debe a la falta de información en el REPS para identificar si el paciente se queda internado en un establecimiento por un tiempo considerable. De igual manera sucede con los minoristas y prestadores de bienes médicos, para quienes la información no es suficiente para clasificarlos dentro de esa categoría.

En síntesis, los resultados permiten conocer cómo se distribuye la prestación de servicios de salud en el país, donde la mayor participación medida por el número de IPS la tienen los prestadores de atención ambulatoria. Posteriormente a esta clasificación, es importante poder relacionar otro tipo de variables financieras como activos y pasivos que permitan identificar no solo como se distribuyen los prestadores de acuerdo con sus servicios, sino también conocer la participación o el tamaño de estas entidades.

$\mathrm{Al}$ cruzar los datos financieros provenientes de los balances a julio de 2014, correspondientes a los reportes de las IPS de circular única (por parte de las IPS privadas) y al SIHO se encuentra que existe una tasa de reporte de información financiera del $65.4 \%$, donde los hospitales tienen la tasa de cumplimiento más alta registrada con un $88.2 \%$ (520 IPS reportaron información, pero no lograron ser identificadas en la base de datos del REPS, quizás por desactualización de los códigos con los que reportan información; por ejemplo, cambios en los NITS; solo una de las IPS es pública, las 519 restantes son privadas). Estos datos podrían incrementar el reporte de información a un $71.6 \%$ ). Por tanto, los resultados financieros no corresponden al universo de las IPS en Colombia.

Los resultados financieros agrupados según el SCS muestran activos por doce billones, pasivos por 3.3 billones y un patrimonio de 8.7 billones. Aunque los hospitales solo concentran el $16.6 \%$ de las IPS en el país, explican el $90.5 \%$ de los activos reportados. A diferencia de este caso, los prestadores de atención ambulatoria, los cuales tienen una participación del $66.1 \%$, solo tienen el $9.3 \%$ de los activos totales. Las demás categorías tienen una participación inferior al $1 \%$ en los activos. 
CLASIFICACIÓN DE INSTITUCIONES PRESTADORES DE SERVICIOS DE SALUD SEGÚN EL SISTEMA DE CUENTAS DE LA SALUD DE LA ORGANIZACIÓN PARA LA COOPERACIÓN Y EL DESARROLLO ECONÓMICO: EL CASO DE COLOMBIA

Tabla 3. Clasificación SHA, Reporte y balance general a Junio de 2014. 13 Principales CiUdades (Millones De Pesos) ${ }^{6}$

\begin{tabular}{|c|c|c|c|c|c|c|}
\hline Categoría & Subcategoría & IPS & $\begin{array}{l}\text { IPS con reporte } \\
\text { de balance }\end{array}$ & Activos & Pasivos & Patrimonio \\
\hline \multirow{4}{*}{ HP1: Hospitales } & Total & 382 & 310 & 4536880 & 1339651 & 3197229 \\
\hline & HP1.1. & 118 & 108 & 3130689 & 1064402 & 2066287 \\
\hline & HP1.2. & 13 & 10 & 69679 & 17060 & 52620 \\
\hline & HP.1.3. & 251 & 192 & 1336512 & 258189 & 1078323 \\
\hline \multirow{4}{*}{$\begin{array}{l}\text { HP2: Establecimientos } \\
\text { de atención } \\
\text { medicalizada y } \\
\text { residencial }\end{array}$} & Total & 3 & 3 & 22621 & 2527 & 20093 \\
\hline & HP2.1. & 0 & & & & \\
\hline & HP2.2. & 3 & 3 & 22621 & 2527 & 20093 \\
\hline & HP2.9. & 0 & & & & \\
\hline \multirow{15}{*}{$\begin{array}{l}\text { HP3: Prestadores } \\
\text { de atención } \\
\text { ambulatoria }\end{array}$} & Total & 3180 & 2108 & 599125 & 222571 & 376554 \\
\hline & HP3.1. & 1091 & 743 & 183134 & 25880 & 157254 \\
\hline & HP3.1.1. & 62 & 39 & 141 & 81 & 60 \\
\hline & HP3.1.2. & 9 & 7 & 180599 & 24534 & 156066 \\
\hline & HP3.1.3. & 1020 & 697 & 2394 & 1265 & 1128 \\
\hline & HP3.2. & 680 & 446 & 749 & 393 & 356 \\
\hline & HP3.3. & 826 & 508 & 6716 & 3052 & 3664 \\
\hline & HP3.4. & 235 & 187 & 246164 & 139662 & 106501 \\
\hline & HP3.4.1. & 0 & 0 & & & \\
\hline & HP3.4.2. & 18 & 9 & 9 & 4 & 4 \\
\hline & HP3.4.3. & 162 & 131 & 222192 & 138606 & 83587 \\
\hline & HP3.4.4. & 3 & 3 & 20 & 13 & 6 \\
\hline & HP3.4.9. & 52 & 44 & 23943 & 1039 & 22903 \\
\hline & HP3.5. & 191 & 108 & 655 & 363 & 292 \\
\hline & HP3.6. & 157 & 116 & 161707 & 53220 & 108487 \\
\hline \multirow{4}{*}{$\begin{array}{l}\text { P4: Prestadores de } \\
\text { servicios auxiliares }\end{array}$} & Total & 293 & 197 & 600 & 295 & 305 \\
\hline & HP4.1. & 49 & 20 & 78 & 33 & 45 \\
\hline & HP4.2. & 175 & 125 & 408 & 199 & 209 \\
\hline & HP4.9. & 69 & 52 & 114 & 64 & 50 \\
\hline \multirow{3}{*}{$\begin{array}{l}\text { HP5: Minoristas y } \\
\text { otros prestadores } \\
\text { de bienes médicos }\end{array}$} & Total & 44 & 25 & 77 & 34 & 44 \\
\hline & HP5.1. & 1 & 0 & & & \\
\hline & HP5.2. & 43 & 25 & 77 & 34 & 44 \\
\hline $\begin{array}{l}\text { HP6: Centrosde } \\
\text { promoción y prevención }\end{array}$ & HP6.1. & 587 & 337 & 1631 & 621 & 1010 \\
\hline Total & & 4489 & 2980 & 5160934 & 1565699 & 3595236 \\
\hline
\end{tabular}

Fuente: datos financieros a junio de 2014. Circular Única y Sistema de Información Hospitalario

6 Las trece principales ciudades fueros seleccionadas de acuerdo con la población 2015 del DANE. Incluye: Bogotá, D. C., Medellín, Cali, Barranquilla, Cartagena, Cúcuta, Soledad, Ibagué, Bucaramanga, Soacha, Villavicencio, Santa Marta y Pereira. 
La composición por categorías del SCS del año 2011 para las trece ciudades principales muestra activos por valor de cinco billones, pasivos por 1.6 y un patrimonio de 3.6 billones. Los activos representan el $43 \%$ de los activos nacionales. Los resultados encontrados muestran una tasa de reporte financiero de las IPS para las trece ciudades de $66.4 \%$, muy similar a la tasa nacional. De manera desagregada, se evidencia que los hospitales agrupan el $88 \%$ de los activos y representan el $8.5 \%$ de las IPS de las ciudades principales. Los prestadores de atención ambulatoria participan con el $70.8 \%$ de IPS en la ciudad. En segundo y tercer lugar se ubican los centros de promoción y prevención, y los hospitales, quienes proporcionan el $13.1 \%$ y el $8.5 \%$, respectivamente.

\section{Discusión y conclusiones}

El propósito de este trabajo fue desarrollar un algoritmo de clasificación de IPS para Colombia, usando el marco conceptual del sistema de cuentas de la salud de la OCDE (System of Health Accounts [SHA], por sus siglas en inglés) y tomando como fuente los servicios habilitados a abril de 2015 por todas las IPS registradas en el REPS. Como aplicación se utilizó la clasificación obtenida para describir las cuentas del balance general de las IPS públicas y privadas.

La elección del SCS como metodología para clasificar los prestadores de servicios de salud obedeció a que este tipo de taxonomía es utilizada a escala internacional, y esto permite ser comparable con otros países pertenecientes a la OCDE. Además, este método es flexible y se puede adaptar a diversas condiciones del país.

De acuerdo con las grandes categorías de clasificación de prestadores del SCS del año 2011, en Colombia el $66 \%$ de las IPS son prestadores de atención ambulatoria, el $17 \%$ son hospitales, el $11 \%$ son centros de promoción y prevención y el $6 \%$ son prestadores de servicios auxiliares (por ejemplo, laboratorios).

Cuando se clasifica la información financiera de las IPS privadas reportadas a la Superintendencia de Salud y de las IPS públicas reportadas al SIHO usando el SCS 2011, se encuentra que los activos de los prestadores de atención ambulatoria solo constituyen el $9.3 \%$ del total nacional. Mientras que los hospitales, que solo concentran el $16.6 \%$ de las IPS en el país, tienen el $90.5 \%$ de los activos reportados.

La clasificación de establecimientos de atención medicalizada y residencial tuvo una participación casi inexistente, debido a que la prestación de ese tipo de servicios en el país es muy baja o nula. Además de esto, en este clasificador no se tuvo en cuenta tres clasificaciones adicionales del SCS referenciadas a los prestadores de administración y financiamiento del sistema de salud (la naturaleza de las IPS es de prestación y no administrativa), al resto de la economía (hogares como proveedores de atención médica en el hogar y las demás industrias como prestadores secundarios de servicios de salud) y el resto del mundo (no residentes que proporcionan bienes y servicios de salud para residentes del país). Estas dos últimas clasificaciones no se utilizaron debido a que la naturaleza de los datos no permitió realizar este tipo de clasificaciones.

Hasta el momento, las clasificaciones que han sido utilizadas en el país no introducen el tipo de servicios prestados por los prestadores, categorizándolos de acuerdo con los niveles de atención, medida que, si bien es interesante, no se encuentra disponible para los prestadores privados. 
En el ámbito internacional se han propuesto sistemas de clasificación de prestadores basados en la diferenciación de servicios y productos ofrecidos, la integración entre áreas y la centralización o descentralización de decisiones y actividades (5-6). Aunque este tipo de taxonomías puede ser de gran utilidad analítica, las bases de datos de prestadores en Colombia no tienen el detalle para ser aplicables.

En los Estados Unidos el seguro público Medicare utiliza la taxonomía "Healthcare Provider Taxonomy Code Set". Esta clasificación está diseñada para categorizar de acuerdo con el tipo (especialidad o subespecialidad), clasificación (servicio u ocupación) y especialización del prestador. La clasificación es jerárquica y diferencia entre prestadores individuales y prestadores grupales (7). Ejemplos del nivel individual son anestesiología, dermatología. Ejemplos del nivel grupal son agencias, hospitales, Laboratorios, etcétera. Una característica de este sistema es que los prestadores se autoclasifican y pueden escoger pertenecer a varias categorías, siempre que designen una como primaria. Este sistema podría ser adoptado en Colombia, pero implicaría cambios en la normatividad y en el proceso de habilitación actual.

En este trabajo se adoptó la taxonomía de SCS porque ha sido ampliamente implementado en los países pertenecientes a la OCDE, permitiendo realizar análisis comparativos, tanto en la composición de los prestadores como en la distribución del gasto en el ámbito del sector salud.

En futuros trabajos se podría explorar otras clasificaciones que incluyan aspectos como el tamaño, especialidad o subespecialidad médica (como el sistema usado por Medicare) para profundizar en análisis más detallados.

\section{Referencias bibliográficas}

1. Guzman K. Radiografía de la oferta de servicios de salud en Colombia. Doc Trab sobre Econ Reg CEER. 2014;(202):1-55.

2. Organisation for Economic Co-operation and Development. A system of health accounts [internet] 2000 [acceso: $1 .^{\circ}$ de enero del 2015]. Disponible en: https://www.oecd.org/els/health-systems/1841456. pdf

3. Organización Mundial de la salud (OMS). Guía del productor de cuentas nacionales de salud con aplicaciones para los países de ingresos bajos y medios [internet]. 2005 [acceso: 9 de abril del 2015]. Disponible en: http://www.who.int/iris/ handle/10665/43363

4. Organisation for Economic Co-operation and Development. A system of health accounts 2011 internet]. 2011 [acceso: $10^{\circ}$ de enero del 2015]. Disponible en: http:/www.who.int/health-accounts/ methodology/sha2011.pdf

5. Bazzoli GJ, Shortell SM, Dubbs N, Chan C, Kralovec P. A taxonomy of health networks and systems: bringing order out of chaos. Health Serv Res. 1999;33(6):1683-717.

6. Dubbs NL, Bazzoli GJ, Shortell SM, Kralovec PD. Reexamining organizational configurations: an update, validation, and expansion of the taxonomy of health networks and systems. Health Serv Res. 2004;39(1):207-20.

7. Health care provider taxonomy code set [internet]. 2017. [acceso: 10 de marzo del 2017]. [Aprox. 10 páginas]. Disponible en: http://www.wpc-edi.com/ reference/codelists/healthcare/health-care-provider-taxonomy-code-set/ 